\title{
Towards a Process Model for Agile Transformation in E-government Projects
}

\author{
Hanna Looks ${ }^{1 *}$, Jannik Fangmann ${ }^{2}$, Jörg Thomaschewski ${ }^{2}$, Eva-Maria Schön ${ }^{3}$
}

\author{
${ }^{1}$ Department of Computer Languages and Systems, University of Seville, Seville, SPAIN \\ ${ }^{2}$ Faculty of Technology, University of Applied Sciences Emden/Leer, Emden, GERMANY \\ ${ }^{3}$ Faculty of Business \& Social Sciences, HAW Hamburg, Hamburg, GERMANY \\ *Corresponding Author: hanna.looks@iwt2.org
}

Citation: Looks, H., Fangmann, J., Thomaschewski, J. and Schön, E.-M. (2021). Towards a Process Model for Agile Transformation in Egovernment Projects. Journal of Information Systems Engineering and Management, 6(1), em0132. https://doi.org/10.29333/jisem/9571

\section{ARTICLE INFO}

Published: 16 Jan. 2021

\section{ABSTRACT}

E-government projects in public administration often fail due to lacking communication and unclear requirements. Furthermore, users are usually not involved in the development of digital products. The agile transformation offers a promising approach to improve communication and the affinity to change regarding requirements in e-government projects. In addition, the agile transformation, as a paradigm shift from a planoriented to an user-centered development, enables a high degree of user-orientation. In order to support and measure the agile transformation, a screening instrument was developed in previous research and was adapted to the context of public administration. This screening instrument enables the measurement of the progress in agile transformation using a personality questionnaire. In this paper we define a seven-step prototypical process model for the application of the screening instrument in public administrations. This process model accompanies the use of the screening instrument in seven steps as a recommendation for usage and enables a targeted and continuous improvement in the agile transformation process in the context of public administration authorities.

Keywords: agile, agile transformation, continuous improvement, e-government, process model, public administration, user centered

\section{INTRODUCTION}

The digital transformation has changed how products are developed in a way that digitalization projects require a high degree of user-orientation. In this context, the assessment of Looks et al. (2018) shows, that there are still deficits in e-government projects when it comes to cope with this change. To ensure the development of successful e-government products, public administration authorities need to further integrate all the relevant users in the development processes. In addition, the German e-government Act (Bundesministerium des Innern für Bau und Heimat, 2013) also prescribes certain obligatory deadlines for the implementation of digital services, which necessitates a shorter time-to-market period. Furthermore, the assessment shows, that e-government and digitalization projects most usually fail due to lacking communication and unclear requirements (Looks, et al., 2018).

Many of these challenges can be overcome by agile methods like Scrum (Schwaber \& Sutherland, 2017) and Kanban (Anderson, 2010). This is also investigated in the State of Agile report (VersionOne Inc., 2019). Moreover, Looks et al. (2019) examined the agile transformation in the context of public administration. In this context, agile transformation presents great potential as it represents a paradigm shift from a plan-oriented to a human-oriented approach (Beck, et al., 2001).

The objective of this paper is to further investigate agile transformation processes in e-government projects as well as to outline our research journey in this field. In particular, it is our intention to present our preliminary results by answering the following research questions:

- RQ1: What challenges are posed by the adoption of agile practices in public administration?

In previous research, we have conducted a survey in order to analyze the attitude of employees in public administration towards agile methods and agile practices (Looks, et al., 2018). Furthermore, we have assigned the identified challenges to the dimensions of a screening instrument for agile transformation (Fangmann, 2018; Looks et al., 2019), we developed. This article summarizes the findings and elaborates on next steps.

- RQ2: How can the identified challenges be overcome?

In this article, we outline a process model for the agile transformation in the context of public administration. A process model is defined as a generalized solution, which can be applied to perform a concrete activity. By managing the process and guiding the 
user, a process model can be used to improve communication and understanding, thus facilitating the progress of the concrete activity (Münch et al., 2012). In the context of agile transformation in the public administration sector, the process model describes a way to use the screening instrument for deriving appropriate measures to accelerate and improve the progress of agile transformation.

The remainder of the paper is structured as follows: in the first part of the paper we explain our related work and present a summary of models for measuring agility which can be found in the literature. Furthermore, we explain our research methodology. This involves conducting a survey to assess the current state of project management in public administrations and a description of the screening instrument. In the results we answer the research questions and define the prototypical process model for the application of the screening instrument. Finally, we outline our future research in this paper.

\section{RELATED WORK}

For the development of our screening instrument we have carried out an analysis of related work. Some models for measuring agility can be found in the literature. These will be briefly summarized below.

Sidky et al. (2007) have developed the Agile Adoption Framework, which is based on the principles of the Agile Manifesto (Beck et al., 2001). The framework includes the measurement of agility (Sidky Agile Measurement Index, SAMI), and a process model for introducing agile practices within an organization.

Qumer et al. (2007) provide a maturity model called Agile Adoption and Improvement Model (AAIM). It is an extension for the Agile Software Solution Framework (ASSF) (Qumer and Henderson-Sellers, 2009). The ASSF describes on a high abstraction level an approach for the creation and context related adaptation of an agile software development process.

With the Agile Maturity Map, Packlick (2007) defines a maturity model consisting of the components target areas, goals, acceptance criteria and maturity levels. Goals and their acceptance criteria are presented in the form of user stories.

Patel and Ramachandran (2009) have developed the Agile Maturity Model. The model defines five maturity levels, as well as an administration process based on self-assessment through assessment questions. The maturity levels are used to define goals for the software development process. To achieve the goals, the model shows areas of improvement at each maturity level.

So and Scholl (2009) have developed the Perceptive Agile Measurement questionnaire. The questionnaire measures the perceived agility independent of the concrete, technical tools that are used in software development. The items of the questionnaire measure eight factors: iteration planning, iterative development, continuous integration and testing, stand-up meetings, customer access, customer acceptance tests, retrospectives and co-location.

Benefield (2010) has created a model that distinguishes agile maturity in seven dimensions: automated regression testing, code quality metrics, automated deployment and backout, automated builds and configuration management best practices, interlocked delivery and interface integration testing, Test Driven Development (TDD), performance and scalability testing. Each of these dimensions is assessed on the basis of five levels of maturity.

Yin et al. (2011) have developed the Scrum Maturity Model that is based on five maturity levels. For each level of maturity, concrete objectives and practices are defined which must be implemented by the organization in order to achieve the target.

Fontana et al. (2015) have created the Progressive Outcomes Framework. The framework distinguishes six progressive outcomes: practices, team, deliveries, requirements, product and customer. For each of these outcomes, maturity levels are defined, varying in number between three and four.

Gren et al. (2015) have developed a questionnaire that can be used to extend the Agile Adoption Framework (Sidky et al., 2007). The questionnaire replaces the procedure for analyzing organizational readiness according to Sidky et al. (2007). Compared to the organizational readiness the questionnaire is not used to determine the agile potential, but to investigate the current degree of agility.

The analysis of related work reveals that many models are in an early stage of development and further empirical studies are needed to validate these models. Furthermore, it becomes clear that there is a need for support in agile transformation. So far, we could not find any work that deals with the application of the models for measuring or improving the agile transformation in the context of e-government projects.

\section{RESEARCH METHODOLOGY}

In the following, we describe the methods used to answer the research questions presented in section Introduction.

\section{Survey Study}

To answer the first research question, a survey was created with the aim of assessing the current state of project management in German public administration authorities and identifying optimization potentials through the use of agile methods and agile practices (Looks, et al., 2018). A total of 18 items were used in the survey. Five items assessed the work experience, working environment and project experience of the participants, whereas the remaining 13 items were used to obtain information on the current state of work practices as well as the attitude towards the use of agile methods and agile practices. For example, the survey included questions on topics such as experience with agile methods in public administration, work in a project team, interdisciplinary teams, usable software, involvement of users, collaboration at eye level and failure of projects. 
Table 1. Dimensions of the assessment questions

\begin{tabular}{ll}
\hline Dimension & Explanation regarding the attributes of an agile team \\
\hline Communicative & An agile team should communicate with each other often and directly. \\
\hline Change-affine & An agile team should react to dynamic requirements quickly and with flexibility. \\
\hline Iterative & An agile team should be able to develop the product in several iterations. \\
\hline Team-centered & An agile team should act as a self-organized team and on their own responsibility. \\
\hline Product-driven & An agile team should be responsible for making the product the focal point of the development activities. \\
\hline
\end{tabular}

The target group of the survey consisted of employees of public administration authorities in Germany. A total of 38 participants $(\mathrm{N}=38)$ from the public administration sector took part in the survey. These participants are employees from IT, the administrative departments, as well as various specialist departments.

\section{Analysis by Means of a Screening Instrument}

To answer the second research question, a first version of a screening instrument, based on a questionnaire, is developed by Fangmann (2018). This screening instrument was taken to measure the progress of the agile transformation process in public administration.

Previous to the development of the screening instrument, other models for ascertaining the progress of agile transformation processes were analyzed in a systematic literature review (Fangmann, Erstellung eines Screening-Instruments zur Fortschrittsermittlung in der agilen Transformation, 2018). We described these models in the section Related Work.

The evaluated models are the following:

- Agile Adoption Framework (Sidky, et al., 2007)

- Agile Adoption and Improvement Model (Qumer, et al., 2007)

- Agile Maturity Map (Packlick, 2007)

- Agile Maturity Model (Patel and Ramachandran, 2009)

- Perceptive Agile Measurement (So and Scholl, 2009)

- Benefield's Model (Benefield, 2010)

- Scrum Maturity Model (Yin, et al., 2011)

- Progressive Outcomes Framework (Fontana, et al., 2015)

- Validation of the Agile Adoption Framework by Gren et al. (2015)

In contrast to these models, the developed screening instrument allows for an inclusion of user-specific context in the transformation process. This context can be included in the survey for ascertaining the state of the process and thus be adequately represented in the results. This adaptability enables the use of the instrument in the specific context of public administration, as described in the following.

The aim of the survey is to assess the overall impression of the progress in agile transformation in a concise manner. The results enable the comparability of the current progress among the participants of the study. Based on the results of the survey, deficits and optimization potentials concerning agility can be identified and measures to promote the agile transformation can be derived. The questions in the questionnaire are divided into three sections: demographic questions, assessment questions and weighting questions.

- The demographic questions serve to capture the demographic context of the participants.

- The assessment questions are intended to assess the current state of the agile transformation. This questions group includes 28 items which were assigned to six dimensions (see Table 1).

- The weighting questions are intended to capture the context-specific circumstances of the participants in the agile transformation process. For more details on the survey questionnaire and its constituents, please refer to Fangmann (2018).

In order to make the questionnaire appropriate to the context of public administration, the identified challenges (see section Survey study) were analyzed according to the dimensions of the screening instrument (Looks et al., 2019). The mapping was done in several workshops with the authors. The allocation of the identified challenges in connection with project work in public administration made it possible to derive suitable countermeasures that facilitate the agile transformation in public administration.

\section{RESULTS}

In the following, we will outline our preliminary results concerning our research questions (see section Introduction).

The challenges obstructing the agile transformation process in public administration are identified in the review provided in Looks et al. (2018). These identified challenges were taken as a basis for assessing the applicability of the screening instrument by Fangmann in the context of public administration. This resulted in a preset of the weighting questions which allows for the use of the instrument in this context. To enable a continuous improvement of agility in the context of agile transformation, a 
Table 2. Preset of the weighting questions

\begin{tabular}{ll}
\hline Dimension & Scale value \\
\hline Communicative & Especially important \\
\hline Change-affine & Especially important \\
\hline Product-driven & Important \\
\hline Improvement-oriented & Important \\
\hline Team-centered & Neutral \\
\hline Iterative & Somewhat unimportant \\
\hline
\end{tabular}

recommendation for the application of the screening instrument in public administration is presented (Fangmann et al., 2020). This preliminary process model will then be built upon and validated in future research.

\section{What Challenges are Posed by the Adoption of Agile Practices in Public Administration?}

Assigning the various challenges in the agile transformation to the dimensions of the survey questionnaire has allowed for a better investigation of the impacts of the challenges on different aspects of agility. This assignability of the challenges leads to the conclusion that all aspects included in the questionnaire as dimensions are relevant for the context of public administration. A complete list of the challenges attributed to the individual dimensions can be found in Looks et al. (2019).

The assignment of the challenges to the individual dimensions has shown that the need for action in regard to agile transformation varies greatly among the different dimensions. For instance, the dimension communicative offers immense potential for overcoming the challenges identified in the review conducted by Looks et al. (2018), as it stated a lack of communication as the most common reason why projects in public administration fail. The prevalence of strict, vertical hierarchical structures is the decisive reason for communication deficits and the root cause of the lack of osmotic communication.

The study also found that some dimensions of agility can only be inadequately considered in the transformation processes due to legal provisions and regulations. For example, the creation of service specifications required by the mandatory tendering process makes an iterative development and implementation process without a big-upfront-design very difficult, since the requirements to be implemented must be fully documented in a comprehensive service specification. This negates any agile approach in which the requirements are ascertained and defined in an iterative process (Fangmann, 2018).

The relevance of the dimensions in the context of public administration discussed above was elaborated by Looks et al. (2019) by defining a preset for the weighting questions. This enables the relevance to be properly taken into account when using the screening instrument in the specific context.

The preset of the weighting questions is presented in Table 2.

\section{How can the Identified Challenges be Overcome?}

In the previous section, it was shown that the challenges identified in the review can be assigned to the different dimensions defined in the screening instrument. The preset of the weighting questions provides the additional benefit of clearly showing which dimension of the agile transformation requires the most work and improvement by the public administration authority.

The screening instrument thus offers a promising approach for identifying and overcoming the challenges which the public administration system is facing in the process of agile transformation. This identification is carried out with the help of the questionnaire itself. However, there is no defined process model for overcoming the identified challenges by using the screening instrument.

Defining such a process model can lead to broadening the scope of application of the screening instrument beyond simply showing the state of progress, but to also ensure continuous improvement. This would then enable the challenges in agile transformation, identified in the review, to be overcome.

The following serves as a rough outline of such a process model. This prototypical recommendation for the application of the screening instrument will be evaluated in future research based on case studies in public administration. An overview of the process model is presented in Figure 1. Each of the seven steps of this model will be explained below. 


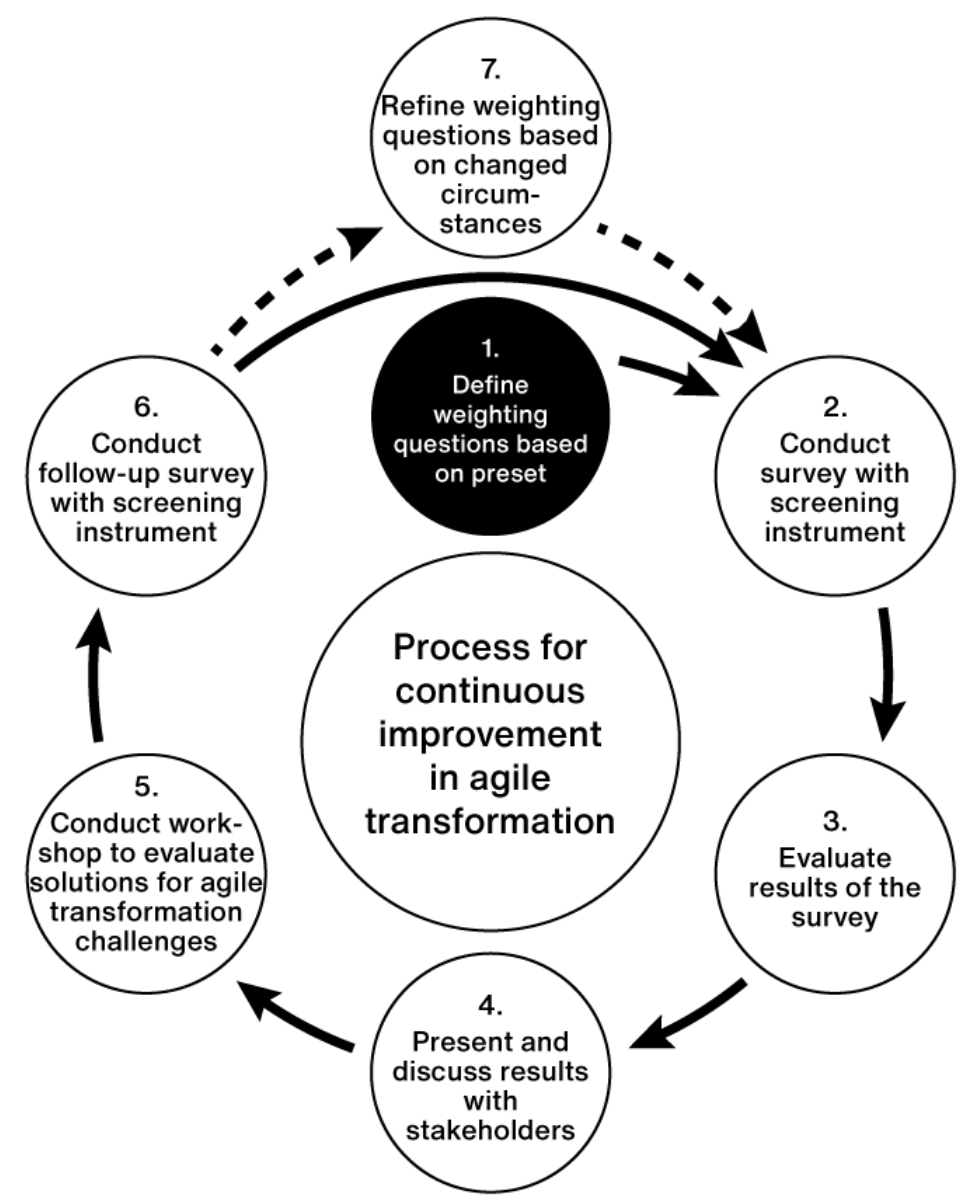

Figure 1. Process for continuous improvement in agile transformation

1. Define weighting questions based on preset: The weighting questions provide an indication regarding the importance and feasibility of the different dimensions of agility. The presented preset of the weighting questions in the context of public administration (Looks et al., 2019) serves as a guideline here. The participating public administration authorities need to define their own weighting systems for the dimensions based on the preset. This step should be guided by an expert who is familiar with the inner workings of the screening instrument. This expert must present the instrument and the purpose of the weighting questions in a way which is comprehensible for the participants. This ensures that sound decisions concerning the weighting of the dimensions are made.

2. Conduct survey with screening instrument: The questionnaire should be completed by all stakeholders who are part of the target group. The target group comprises all persons who participate in the implementation of e-government projects in either an operative or managerial capacity. In addition to the employees from the IT department, this explicitly includes project participants with organizational as well as administrative positions and the relevant specialist departments. This interdisciplinary composition of the target group is intended to deliver a representative overall impression of the progress of the agile transformation. In this part of the process, answering the questionnaire is restricted only to the demographic questions and the assessment questions. The weighting questions will be defined as established in step 1.

3. Evaluate results of the survey: After this step, the results of the survey must be evaluated. The test value determination of the screening instrument (Fangmann, 2018) can be used for this purpose. The value thus determined indicates the progress of the agile transformation process in the public administration authority being examined. The report on this test value determination is comprised of an introduction, overview, result and benchmark. The introduction serves to inform the participants about the goal and content of the survey. The overview section is to briefly present a summary of the results for the individual dimensions. The result section provides the detailed results for the individual dimensions in a tabular form. The benchmark section will present a comparison of the results of the administration authority with other participants of the survey. A detailed explanation as well as examples of application of the test value determination method can be found in Fangmann (2018).

4. Present and discuss results with stakeholders: Once the test value has been determined, the results of the survey must be presented to the relevant stakeholders in a clear and understandable manner. It is recommended to discuss the results in the context of a meeting led by the expert who is accompanying the agile transformation process (see step 1). The goal is to reach a common understanding of the results.

5. Conduct workshop to evaluate solutions for agile transformation challenges: It is recommended to hold a workshop as the next step in which the challenges and possible measures for overcoming them are to be discussed and reflected. The challenges to the agile transformation result from the examination of the results for the individual dimensions. The 
participants in the workshop are to work on defining measures to combat these challenges, for which purpose various agile practices and methodologies can be discussed as possible solutions for these challenges. In a subsequent step, suitable measures for assuring continuous improvement are to be defined for the dimensions. To enable a verification of the success of these measures, it is recommended to define and set a clear target test value to be reached in the next test value determination.

6. Conduct follow-up survey with screening instrument: A follow-up survey can be used as a tool for determining the achievement of the defined goals. For this purpose, the process model for the application of the screening instrument can be followed again starting with step 2. The weighting questions must not be changed from the previous survey to ensure the comparability of the results.

7. Refine weighting questions based on changed circumstances: After such comparison has be completed, it is possible to adapt the weighting questions to better reflect the lessons learned and the changed circumstances. In doing so, it is important to remember that such change would have an impact on the result thus obtained. It is therefore necessary to determine a new result with the answers to the assessment questions from the current survey as well as the changed weighting questions. This result then serves as the basis for comparison and determining the success with the subsequent survey. This step is optional and can be omitted if no adaption of the weighting questions is needed.

\section{FUTURE RESEARCH}

This paper provides an initial outline of a process model for applying the screening instrument in the context of public administration.

A seven-step process model for the application of the screening instrument was presented which shall be revisited and improved in future research. This process model should include an identification of concrete methods and practices for resolving the identified challenges to ensure that the agile transformation process keeps moving forward.

In the course of developing the model, the following questions arose which shall be investigated in more detail in future research:

- What are the specific prerequisites that the public administration authority must meet in order to use the screening instrument?

- Should the weighting questions be uniformly defined for the entire public administration authority or individually for the various participating teams?

- Do the content and scope of the test value determination need to be expanded for the comparability of the results of the individual measurements?

- Is it necessary to adapt the assessment questions with regard to the context of public administration?

Once these questions are answered, and after the suggested process model has been properly configured, it must be evaluated on the basis of case studies and expert validations. The challenges identified by Looks et al. (2018) concern the public administration system in Germany. A review for the public administration system in Spain, which is to be published in the near future, will provide a sound basis for an international comparison.

\section{CONCLUSION}

In this article we have outlined the current state of our research regarding the measurement of the progress of agile transformation in public administration. This overview included a discussion of the challenges when adapting agile practices and methodologies (RQ1) and also the presentation of a possible way to overcome these challenges, which is the presented process model (RQ2).

To answer the first research question, a review was conducted by Looks et al. (2018) which provided an overview of the current state of the adaptation of agile practices in public administration. The survey provided information both on the views taken by the participants on the subject of agile work as well as the challenges in the adaptation of the agile paradigm. The assignment of the identified challenges to the various dimensions of the screening instrument by Fangmann (2018) resulted in the preset for the weighting questions (Looks et al., 2019), which reflects the relevance of the individual dimensions of the screening instrument in the context of public administration.

In regard to the second research question, a seven-step process model for the application of the screening instrument by Fangmann (2018) in the context of public administration was defined. It is intended to establish a mindset for an agile form of work in public administration with a continuous process of improvement.

The process model is to be understood as an abstract prototype which must be further developed and improved in future research. Furthermore, several open questions arose during the process of developing this recommendation, which are presented in the section Future Research. Finding adequate answers for these questions and defining concrete practices and methods for the individual steps can serve to further improve the process model, and thus to support a targeted agile transformation in the public administration sector. By implementing these steps, we wish to continue our research in the field of agile transformation in egovernment projects with the help of additional international research projects. 


\section{REFERENCES}

Anderson, D. J. (2010). Kanban: Successful Evolutionary Change for Your Technology Business. Sequim, Washington: Blue Hole Press.

Beck, K., et al. (2001). Agile Manifesto. Available at: https://agilemanifesto.org (Accessed: 20 July, 2020).

Benefield, R. (2010). Seven Dimensions of Agile Maturity in the Global Enterprise: A Case Study. In 2010 43rd Hawaii International Conference on System Sciences, IEEE Xplore. https://doi.org/10.1109/HICSS.2010.337

Bundesministerium des Innern für Bau und Heimat (2013). Übersicht zu Umsetzungsverpflichtungen aus dem E-Government-Gesetz [Overview of implementation obligations from the E-Government Act]. Available at: www.bmi.bund.de/SharedDocs/downloads/ DE/veroeffentlichungen/themen/moderne-verwaltung/umsetzungsverpflichtung-egov.pdf (Accessed: 20 July, 2020).

Fangmann, J. (2018). Erstellung eines Screening-Instruments zur Fortschrittsermittlung in der agilen Transformation [Creation of a screening instrument to determine progress in the agile transformation] (Masters Thesis), University of Applied Sciences, Emden/Leer.

Fangmann, J., Looks, H., Thomaschewski, J. and Schön, E.-M. (2020). Agile transformation in e-government projects. In 202015 th Iberian Conference on Information Systems and Technologies (CISTI), IEEE Xplore. https://doi.org/10.23919/CISTI49556.2020.9141094

Fontana, R. M., Meyer, V., Reinehr, S. and Malucelli, A. (2015). Progressive Outcomes: A framework for maturing in agile software development. Journal of Systems and Software, 102, 88-108. https://doi.org/10.1016/j.jss.2014.12.032

Gren, L., Torkar, R. \& Feldt, R. (2015). The prospects of a quantitative measurement of agility: A validation study on an agile maturity model. Journal of Systems and Software, 107, 38-49. https://doi.org/10.1016/j.jss.2015.05.008

Looks, H., Fangmann, J., Thomaschewski, J. and Schön, E.-M. (2019). Agilität und Nutzerzentrierung in der öffentlichen Verwaltung. In Mensch und Computer 2019 - Usability Professionals, Gesellschaft für Informatik e.V. Und German UPA e.V. https://doi.org/10.18420/muc2019-up-0301

Looks, H., Schön, E.-M. and Thomaschewski, J. (2018). Agile Projekte in öffentlichen Verwaltungen - Eine Bestandsaufnahme. In Mensch und Computer 2018 - Usability Professionals, Gesellschaft für Informatik e.V. Und German UPA e.V. https://doi.org/10.18420/muc2018-up-0156

Münch, J., Armbrust, O., Kowalczyk, M. and Soto, M. (2012). Software Process Definition and Management. Berlin, Heidelberg: Springer Berlin Heidelberg. https://doi.org/10.1007/978-3-642-24291-5

Packlick, J. (2007). The Agile Maturity Map A Goal Oriented Approach to Agile Improvement. In Agile 2007 (AGILE 2007), IEEE Xplore. https://doi.org/10.1109/AGILE.2007.55

Patel, C. and Ramachandran, M. (2009). Agile Maturity Model (AMM): A Software Process Improvement framework for Agile Software Development Practices. International Journal of Software Engineering, 2(1), 3-28.

Qumer, A. and Henderson-Sellers, B. (2009). Agile Software Solution Framework: An Analysis of Practitioners' Perspectives. In Information Systems: Modeling, Development, and Integration. Springer Berlin Heidelberg. https://doi.org/10.1007/978-3-64201112-2_6

Qumer, A., Henderson-Sellers, B. and Mcbride, T. (2007). Agile adoption and improvement model. Proceedings of the European and Mediterranean Conference on Information Systems, EMCIS 2007.

Schwaber, K. and Sutherland, J. (2017). The Scrum Guide. Available at: https://www.scrumguides.org/docs/scrumguide/v2017/ 2017-Scrum-Guide-US.pdf (Accessed 20 July, 2020).

Sidky, A., Arthur, J. and Bohner, S. (2007). A disciplined approach to adopting agile practices: the agile adoption framework. Innovations in Systems and Software Engineering, 3(3), 203-216. https://doi.org/10.1007/s11334-007-0026-z

So, C. and Scholl, W. (2009). Perceptive Agile Measurement: New Instruments for Quantitative Studies in the Pursuit of the SocialPsychological Effect of Agile Practices. Berlin, Heidelberg, Springer Berlin Heidelberg. https://doi.org/10.1007/978-3-642-018534_11

VersionOne Inc. (2019). 13th Annual State of Agile Report. Available at: https://stateofagile.com/\#ufh-i-613553418-13th-annualstate-of-agile-report/7027494 (Accessed 20 July, 2020).

Yin, A., Figueiredo, S. and Silva, M. (2011). Scrum Maturity Model. ICSEA 2011. https://doi.org/10.1061/9780784411773 